\title{
Empirical Correlation of the Morphology of Coiled Carbon Nanotubes with Their Response to Axial Compression
}

\author{
Jabulani R. Barber, ${ }^{1}$ Jeffrey S. Boyles, ${ }^{1}$ Aldo A. Ferri, ${ }^{2}$ and Lawrence A. Bottomley ${ }^{1}$ \\ ${ }^{1}$ Georgia Institute of Technology, School of Chemistry and Biochemistry, Atlanta, GA 30332-0400, USA \\ ${ }^{2}$ Georgia Institute of Technology, The George W. Woodruff School of Mechanical Engineering, Atlanta, GA 30332-0405, USA \\ Correspondence should be addressed to Lawrence A. Bottomley; bottomley@gatech.edu
}

Received 23 July 2013; Accepted 23 October 2013; Published 2 February 2014

Academic Editor: Paresh Chandra Ray

Copyright (C) 2014 Jabulani R. Barber et al. This is an open access article distributed under the Creative Commons Attribution License, which permits unrestricted use, distribution, and reproduction in any medium, provided the original work is properly cited.

\begin{abstract}
The mechanical response of thirteen different helical multi-walled carbon nanocoils to axial compression is reported. Each nanocoil was attached to the apex of a cantilever probe tip; its dimensions and orientation relative to the tip apex were determined with scanning electron microscopy. The atomic force microscope was employed to apply a cyclic axial load on the nanocoil. Its mechanical response was determined by simultaneous collection of the thermal resonance frequency, displacement, and oscillation amplitude of the cantilever-nanotube system in real time. Depending upon compression parameters, each coil underwent buckling, bending, and slip-stick motion. Characteristic features in the thermal resonance spectrum and in the force and oscillation amplitude curves for each of these responses to induced stress are presented. Following compression studies, the structure and morphology of each nanocoil were determined by transmission electron microscopy. The compression stiffness of each nanocoil was estimated from the resonant frequency of the cantilever at the point of contact with the substrate surface. From this value, the elastic modulus of the nanocoil was computed and correlated with the coiled carbon nanotube's morphology.
\end{abstract}

\section{Introduction}

Carbon nanotubes have interesting structural diversity. They can be hundreds of microns long yet have diameters of only a few nanometers. Single-walled carbon nanotubes are generally straight whereas multiwalled carbon nanotubes can have straight as well as coiled morphologies. The synthesis and characterization of coiled carbon nanotubes (CCNTs) were first reported two decades ago $[1,2]$. Since that time, a great deal of effort has been expended in improving the yield and quality of CCNTs [3-7] and in characterization of their mechanical properties [8-12]. Applications of CCNTs as components in composites [13-19] and devices [3, 20, 21] have emerged.

The objectives of the work presented herein were to determine the stiffness of CCNTs to axial compression and to correlate this stiffness with their composition/structure. Our approach involves attaching an individual nanotube to the tip of an atomic force microscope (AFM) probe and then using this instrument to apply a controlled mechanical load to the nanocoil [22-24]. Mechanical loading of straight or coiled nanotubes results in compression, buckling, and sliding on the substrate surface $[9,22,23,25-32]$. To meet our objective, thirteen CCNT-modified cantilever probes were prepared. The geometry of each CCNT was first determined by SEM imaging, then its mechanical response to compressive loading was monitored by multidimensional force spectroscopy (MDFS), and finally its chemical structure was determined by transmission electron microscope (TEM) imaging. This sequence was mandated by the fragility of the CCNTs to electron beam bombardment.

MDFS refers to the simultaneous collection of the thermal resonance frequency, displacement, and oscillation amplitude of the cantilever. From analysis of MDFS of cantilever deflection and thermal resonance data through the compression/decompression cycle on the CCNT, we have identified thermal resonance vibrational signatures for buckling, bending, and slip-stick events. We have also acquired new information concerning interactions of the nanocoil with the substrate surface. The outcomes of this effort 
include the following: (i) a more thorough analysis of the nanocoil's response to mechanical compressive loading, (ii) a demonstration of the advantages of MDFS over conventional techniques (i.e., shifts in oscillation amplitude and phase), and (iii) a correlation of carbon nanospring stiffness with composition.

\section{Experimental}

The thirteen CCNTs used herein were fabricated and mounted on AFM probes (MikroMasch, Wilsonville, OR) at NASA Ames Research Center. The CSC-38 chips have three beams. In most cases, the carbon nanocoil was attached to the longest of the cantilevers having an average stiffness of $0.03 \mathrm{~N} / \mathrm{m}$. Only one was modified per chip. The rationale behind this is the following: (1) the field of view of the optical microscope used to fabricate these probes enabled viewing of only one cantilever at a time; (2) our AFM is capable of tracking only one cantilever at a time; and (3) compression studies on a nanocoil attached to a short cantilever would subject the nanocoil attached to a longer cantilever to such high compressive loads that mechanical damage is highly likely. The nanocoil was prepared via CVD methods and attached to the cantilever probe tip through a manual attachment technique. This process is explained in detail elsewhere [33].

Characterization of the placement and orientation of the CCNTs was performed using a LEO model 1550 SEM at a resolution of $2 \mathrm{~nm}$. Characterization of the CCNT structure and morphology was performed using a JEOL100CX-2 TEM. A custom holder capable of securing the entire cantilever chip within the beam path was designed and manufactured. Initial TEM imaging of the CCNT-modified cantilever tips revealed beam-induced damage to the nanocoil. We speculate that damage occurred because there is no direct path for heat to dissipate from the sample to the holder. To reduce damage to the nanocoil, TEM imaging was postponed until after completion of compression studies and the duration of direct beam contact with the nanocoil was kept to a minimum.

All compression tests were performed under a nitrogen atmosphere using a Nanoscope IIIa (Veeco, Santa Barbara, CA) scanning probe microscope operating in Tapping Mode. The piezo scanner was calibrated in $x, y$, and $z$ dimensions using NIST certified calibration gratings (MikroMasch). The substrate was a template-stripped gold surface [34] that was attached to a metal shim using a heat-cured epoxy (Epotek, Billerica, MA). The gold surface was then treated with 11-dodecanethiol (Sigma-Aldrich) to minimize adhesion of the nanocoil to the substrate. In addition to standard shims, a set of angled shims were fabricated with $5^{\circ}$ and $10^{\circ}$ slopes [35]. Detector sensitivity was determined by deflecting the cantilever under conventional AFM operation without making contact to the CCNT. This was achieved using a micromachined silicon pillar specially fabricated for this purpose.

The microscope was set in force curve acquisition mode and the scanner was cycled in the $z$-direction for $\sim 2$ hours without allowing the nanocoil to make contact with the substrate. Next, oscillation of the cantilever was initiated at a frequency just below its natural resonance. The oscillation amplitude was monitored as the scanner extension start position was raised to the point where the nanocoil made contact with the substrate. Then cantilever deflection and oscillation amplitude were monitored as a function of scanner vertical movement. The range of scanner motion was held constant at $2.5 \mu \mathrm{m}$; the initial scanner position was adjusted to set the desired extent of compression on the nanocoil. Force and oscillation amplitude curves were acquired at a scanner velocity of $100 \mathrm{~nm}$ per sec with and without external excitation of the cantilever.

Thermal resonance data was acquired by routing the cantilever deflection signal from the AFM signal access module to a BNC-2110 connector block into the computer via a PCI6120 data acquisition card (DAQ) (National Instruments, Austin, TX). The DAQ was also used to acquire the timedependent deflection data, piezo drive voltage signal, and the horizontal deflection signal. The horizontal cantilever deflection signal was accessed directly from the printed circuit board on the base of the microscope and connected to the connector block. Data acquisition software written in LabVIEW (National Instruments, Austin, TX) converted the deflection data into the frequency domain using a BlackmanHarris window. Cantilever resonance data was acquired with a sampling rate of $800 \mathrm{kHz}$; the resolution along the frequency axis of the power spectral density (PSD) trace was $57 \mathrm{~Hz}$. To improve the signal-to-noise ratio, an ensemble average of fifteen FFTs was taken. Thus, fifty ensemble-averaged PSD traces were recorded during each force curve.

Cantilever spring constants and Q-factors were computed using a data processing program that was written in MATLAB (MathWorks, Natick, MA). The program is based on the method of Hutter and Bechhoefer [36] for calculating spring constants and Q-factors employing a nonlinear least squares fitting of thermal resonant spectral data.

\section{Results}

Prior to axial compression of each of the multiwalled CCNTs in the AFM, their placement and orientation on the probe were determined by SEM. Figure 1 displays SEMs of one of the nanocoils used in this study; this nanocoil was mounted onto a CSC-38 silicon cantilever with a spring constant of $0.037 \mathrm{~N} / \mathrm{m}$. SEM images acquired from several views were analyzed to provide measures of the coil diameter $(D)$, nanotube diameter $(d)$, free length $\left(l_{0}\right)$, and number of active coils $(n)$ extending from the probe tip. The contact angle was estimated from the known tilt of the cantilever in the holder and from measurement of the relative angle of the nanocoil to the probe tip as determined from the SEMs taken at orthogonal views. From these, the compression angle of the nanocoil ( $\left.\alpha_{\text {comp }}\right)$ was determined. Details concerning the orientation and geometry of the CCNTs used in this study are presented in Table 1.

When the probes were mounted in the AFM, extension of the $z$-piezo scanner supplied mechanical loading to the CCNT. The magnitude of applied load is a function of scanner 
TABLE 1: Summary of CCNT geometries from SEM imaging.

\begin{tabular}{|c|c|c|c|c|c|}
\hline $\begin{array}{l}\text { CCNT } \\
\text { no. }\end{array}$ & Chip/cantilever & No. of coils & $\begin{array}{c}\text { Free length } \\
(\mu \mathrm{m})\end{array}$ & $\begin{array}{c}\text { Angle from } \\
\text { tip normal }\left({ }^{\circ}\right)\end{array}$ & $\begin{array}{c}\text { Compression } \\
\text { angle }\left({ }^{\circ}\right)\end{array}$ \\
\hline 1 & CSC-38/C & 2 & 1.40 & 16.8 & 4.8 \\
\hline 2 & CSC-38/B & 9 & 4.01 & 5.9 & 6.1 \\
\hline 3 & CSC-38/B & 6 & 6.00 & 7.3 & 4.7 \\
\hline 4 & CSC-38/B & 4 & 5.17 & 19.0 & 7.0 \\
\hline 5 & CSC-38/B & 4.5 & 11.26 & 7.58 & 4.4 \\
\hline 6 & CSC-38/B & 3 & 9.04 & 5.57 & 6.4 \\
\hline 7 & CSC-38/B & 4 & 5.08 & 24.1 & 12.1 \\
\hline 8 & CSC-38/C & 2.5 & 7.93 & 4.37 & 7.6 \\
\hline 9 & CSC-38/C & 12 & 11.95 & 12.0 & 0.04 \\
\hline 10 & CSC-38/C & 3.75 & 6.72 & 6.11 & 5.9 \\
\hline 11 & CSC-38/C & 2.75 & 3.47 & 10.5 & 1.4 \\
\hline 12 & CSC-38/C & 13 & 7.07 & 40.7 & 28.7 \\
\hline 13 & CSC-38/C & 2 & 6.60 & 20.7 & 8.7 \\
\hline
\end{tabular}

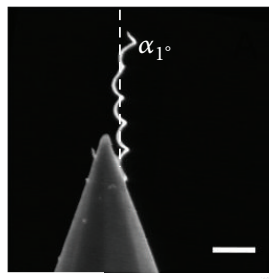

(a)

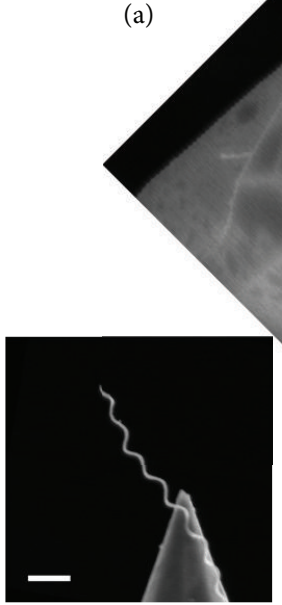

(c)

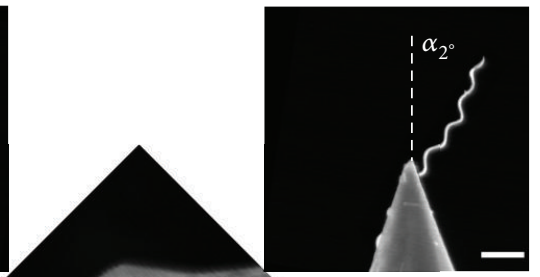

(b)
IGURE 1: SEM images of one of the CCNT-modified AFM probes from orthogonal views: downbeam (a), right side (b), left side (c), upbeam (d), and top (e). Scale bars represent $2 \mu \mathrm{m}$, unless otherwise noted.

extension following contact of the nanocoil with the substrate times the spring constant of the cantilever to which the nanocoil is attached. In this study, to systematically vary the applied load, the scanner start position was varied; the range and speed of scanner extension were kept constant at $2500 \mathrm{~nm}$ and $0.02 \mathrm{~Hz}$, respectively.

To reduce the probability of mechanically induced damage and/or detachment of the nanocoil, compression/decompression studies were performed without contacting the silicon probe tip to the surface. This constraint, coupled with the desire to precisely control the magnitude of applied load, necessitated the following protocol. Prior to approaching the surface, the microscope was first engaged in tapping mode with the cantilever driven at its fundamental vibrational frequency. The microscope was then switched to force curve mode and the cantilever oscillation amplitude was used to determine the point of contact between the nanocoil and the surface. Contact was indicated by a sharp decrease in the oscillation amplitude. Setting the oscillation drive amplitude to zero enabled monitoring of the thermal resonance of the cantilever continuously during cyclic movement of the scanner. Thus, at each compression increment, force curves, oscillation amplitude plots, and thermal resonance spectra were acquired.

Figure 2 presents the thermal resonance frequency as a function of time over four cycles of scanner approach and retraction as the CCNT (Figure 1) was brought into and out of contact with an 11-dodecanethiol-modified templatestripped gold substrate surface anchored onto a flat shim. In this figure, the CCNT was in contact with the substrate only during the last $250 \mathrm{~nm}$ of scanner extension. Two thermal resonance frequencies are observed over the frequency band of 0 to $400 \mathrm{kHz}$. Prior to contact, the fundamental cantilever resonance frequency (orange) is found at $9.4 \mathrm{kHz}$ and the secondary vibrational mode (blue) is observed at $71.7 \mathrm{kHz}$. These frequencies reflect the free vibration of the cantilever since the effective mass of the nanocoil is negligible. At the point of contact, the nanocoil becomes pinned on the substrate and the thermal resonance rises to $30 \mathrm{kHz}$ reflecting the stiffness of the nanocoil attached to the cantilever probe. The CCNT begins buckling shortly after contact with the surface and the fundamental resonance frequency drops from $30 \mathrm{kHz}$ to $12 \mathrm{kHz}$ at full extension of the scanner. During the retract portion of the scan cycle, the resonance frequency increases until the point of release of the nanocoil from the surface. At this point, the resonance frequency returns to its fundamental resonance value in free space $(9.4 \mathrm{kHz})$. A similar trend was observed for the secondary vibrational mode of the cantilever. 


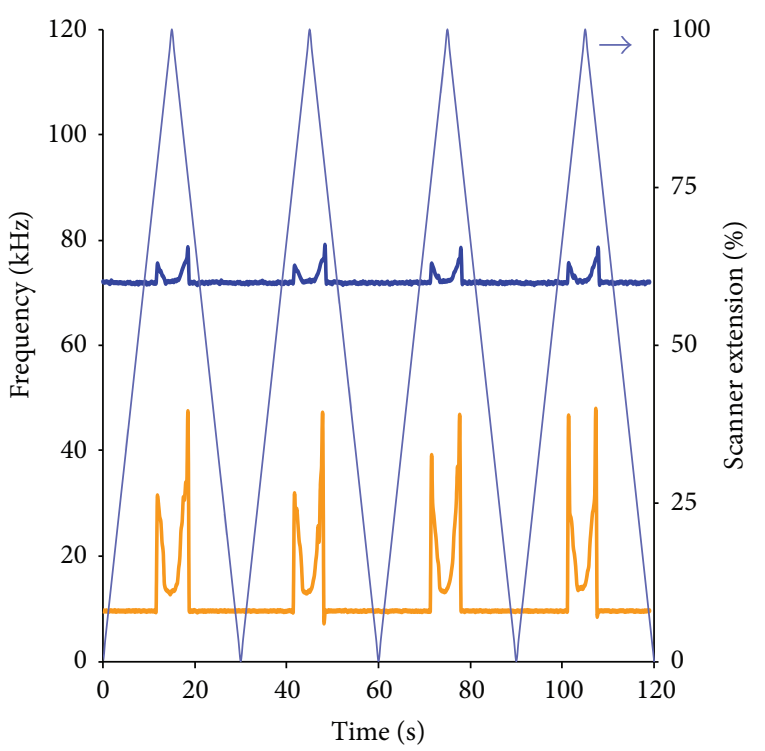

FIGURE 2: Thermal resonance data of the CCNT-modified cantilever during cyclic compressions applied via AFM. During each cycle, the CCNT is compressed to a distance of $250 \mathrm{~nm}$. The $\mathrm{z}$-piezo scanner extension was $2500 \mathrm{~nm}$. The cyclical movement of the scanner with time is identified by the light blue trace.

A salient feature of the data presented in Figure 2 is the reproducibility of the frequency shifts from one scanner cycle to the next. In each cycle there is an asymmetry in the frequency response of the system; higher values are observed during retract of the scanner (i.e., $30 \mathrm{kHz}$ ) than on approach $(42 \mathrm{kHz})$. A plausible explanation for this observed hysteresis is increased tension caused by adhesive interactions between the coil and the surface $[37,38]$.

Figure 3(a) displays the corresponding force curve acquired by the AFM during one scanner cycle for the CCNT under discussion (Figure 1). The point of contact on the approach portion of the force curve occurred at the same time point as the jump in frequency presented in Figure 2. Similarly, the return of the frequency to $9.4 \mathrm{kHz}$ occurred at precisely the same time as the release event in the retract portion of the force curve. The downward deflection of the cantilever on scanner retraction is consistent with a small amount of adhesion between the nanocoil and the chemically modified surface. An adhesion force of $1.6 \pm 0.3 \mathrm{nN}$ was computed from the measured downward deflection of the system at the point of release.

Figure 3 also presents force curves acquired at different compression distances between the CCNT and the substrate surface after first contact. The range in compression distances examined spanned from 125 to $875 \mathrm{~nm}$. Salient features in the approach curves over this distance interval include two regions of increasing cantilever deflection with scanner extension and an intermediate region where no deflection is occurring despite upward movement of the substrate. Note that the second linear region is not due to contact with the probe since the nominal length of the nanocoil is $4.7 \mu \mathrm{m}$ (helical pitch of $1.16 \mu \mathrm{m}$ ) which is more than thrice the distance the scanner extends following contact.

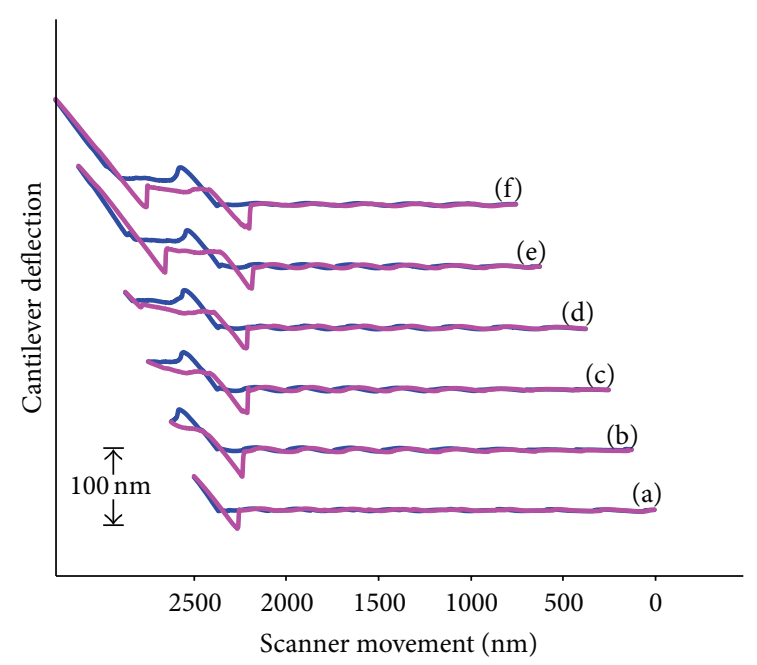

FIGURE 3: Force curve data collected during compression of the CCNT at an approach angle of $7^{\circ}$ to the surface. The total $z$ piezo scanner size was held constant at $2500 \mathrm{~nm}$. The compression amounts on the nanocoil were increased by changing the start position of the scanner cycle. The force curves are collected at (a) 125 , (b) 250 , (c) 375 , (d) 500, (e) 750, and (f) $875 \mathrm{~nm}$ of compression distance. The data are offset so that the jump to contact in the approach curves (blue) is aligned for comparison. Magenta traces denote retract curves.

The oscillations present in regions of the force curves where the CCNT is not in contact with the substrate surface are a result of optical interference from laser light reflecting off both the cantilever and the substrate. This interference is also detected as a periodic fluctuation of noise floor in the thermal resonance (data not shown).

Figure 4 presents the frequency response of the CCNT during the compression events depicted in Figure 3. To assist the reader in comparing the frequency response as a function of time and contact distances, the traces have been offset along the frequency axis. The scanner extension data is also presented in a secondary $y$-axis to enable the reader to visualize the time point of scanner retraction facilitating comparison of the frequency response with corresponding force curves in Figure 3. Traces (i)-(iii) in Figure 4 are comparable in shape to those reported previously for a nanocoil affixed to a stiff cantilever [22]. Traces (iv)-(vi) exhibit a second shift in frequency for both resonance modes; this feature was not previously observed. Note that the discontinuity in the traces for the primary resonance mode reflects a region where the resonance amplitude decreased in magnitude until it was no longer distinguishable from the background. Also note that during approach, the time point at which a second rise in frequency occurs corresponds to the time at which the cantilever bends upward for the second time in the force curves presented in Figure 3.

Figure 5 displays the secondary mode frequency response (a), the force curve (b), and oscillation amplitude (c), data collected during a single scanner cycle for a contact distance of $875 \mathrm{~nm}$ and stacked in a manner to aid comparison. Note that in this figure, the frequency, cantilever deflection, 


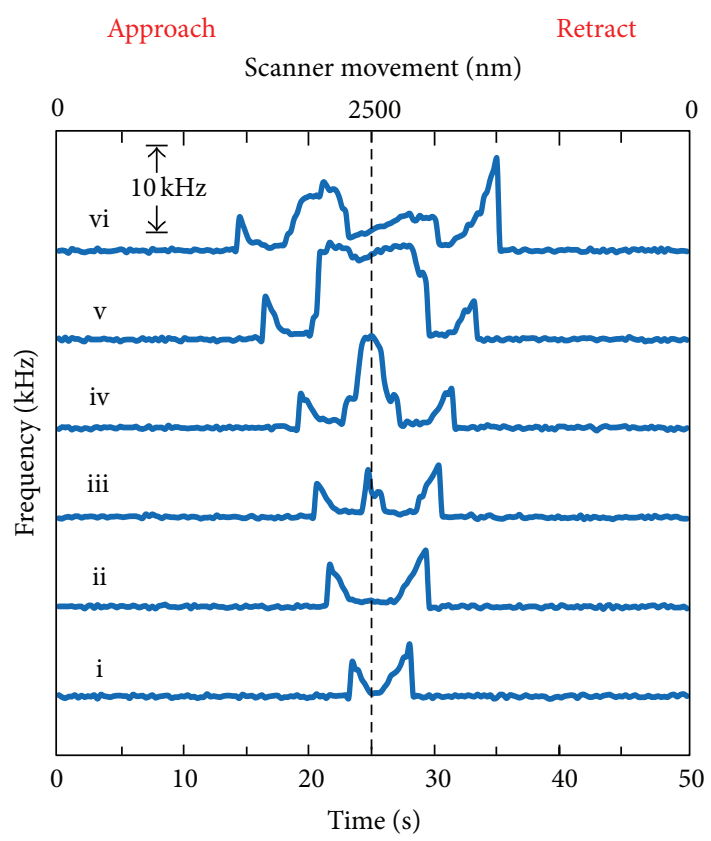

(a)

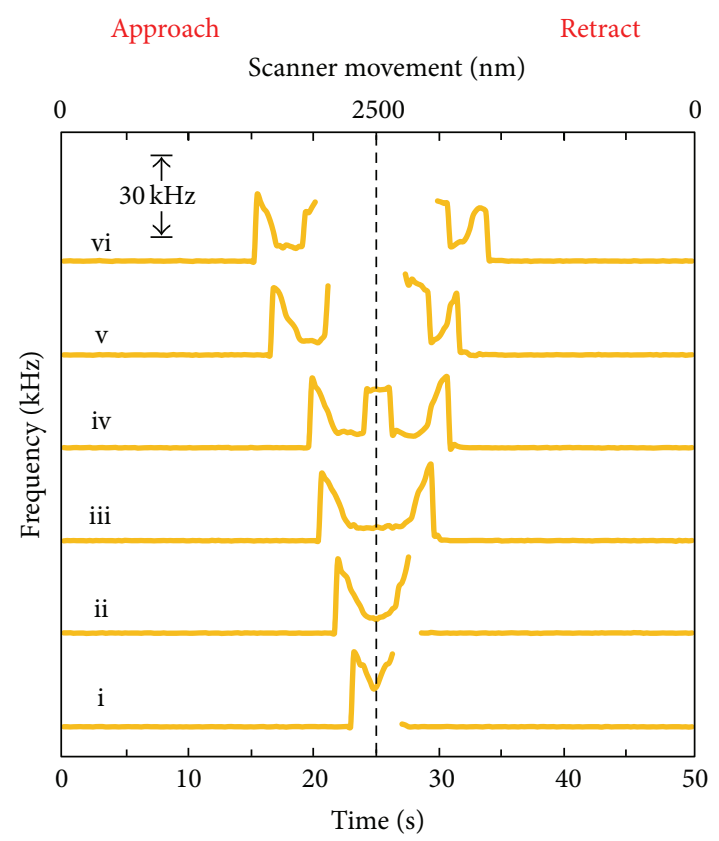

(b)

FIGURE 4: Thermal resonance frequency response of the CCNT at varying compression conditions. The $z$-piezo scan size was set at $2500 \mathrm{~nm}$ for the (b) primary and (a) secondary resonance modes at the following extent of compression: (i) 125, (ii) 250, (iii) 375, (iv) 500, (v) 750, and (vi) $875 \mathrm{~nm}$ are presented. The occurrence of slip stick is observed beginning at a compression distance of approximately $457 \mathrm{~nm}$ and can be observed at larger compression distances.

and change in oscillation amplitude data are plotted as a function of scanner movement. The cantilever-CCNT system remains in free space vibration until the scanner extends to the point of contact between the nanocoil and the substrate. The initial contact (designated with arrow \#1 in all three panels) occurs at $\sim 1620 \mathrm{~nm}$ into the scan cycle and is marked by a decrease in cantilever oscillation amplitude, upward deflection of the cantilever, and an increase in its resonant frequency when the cantilever is driven by only thermal motion. As the scanner continues to extend, there is a nonlinear drop in frequency simultaneously with a linear upward deflection of the cantilever (designated with arrow \#2). This combined behavior is consistent with CCNT buckling. At $\sim 1815 \mathrm{~nm}$ of scanner extension, the cantilever begins to deflect downward, towards the substrate. Beginning at $\sim 1865 \mathrm{~nm}$ of scanner extension (designated with arrow \#3), cantilever deflection and resonant frequency remain constant even though the scanner is continuing to extend towards the cantilever. Constant deflection and resonant frequency are observed until the scanner has extended to $\sim 2150 \mathrm{~nm}$ (designated with arrow \#4). At this point, the cantilever bends upward in a linear fashion and its resonant frequency increases with scanner extension until the point at which the scanner begins to retract. As the scanner retracts, the expected decrease in cantilever deflection and resonant frequency is observed. At a scanner position of $\sim 2105 \mathrm{~nm}$ on the retract direction, a sudden upward motion of the cantilever is observed to be typical of sudden release of a portion of the CCNT from the substrate surface (designated with arrow \#5). The resonant frequency and deflection remain constant until the scanner has retracted to $1450 \mathrm{~nm}$. Then, the nanocoil detaches from the surface (designated with arrow \#6) and the frequency of the cantilever-nanocoil system returns to its free space value.

Three possible explanations for the behavior described above are that the CCNT undergoes (1) postbuckling, a deformation of the wall structure of the nanotube [39], (2) higher order buckling that deforms the helical structure of the nanocoil, or (3) movement across the substrate by slipstick motion [40]. Two "ringing" events are observed during retract motion of the scanner in the oscillation amplitude plot (Figure 5(c)) indicated by \#5 and \#6. During scanner retraction, tension increases until the restorative forces acting on the cantilever overcome the adhesive interaction. A sudden release from contact causes a short-lived oscillation of the cantilever, that is, "ringing." The observation of two "ringing" events in the oscillation amplitude plot is supportive of the slip-stick hypothesis. We postulate that slip-stick motion results in a larger portion of the nanocoil coming into contact with the substrate. The tension imposed by scanner retraction and the increased contact area should result in a larger resistance to the restorative force of the cantilever. Release of a portion of the CCNT completely from the surface results in ringing as the coil adopts a new geometry.

To test the slip-stick hypothesis, we systematically varied the nanocoil angle at point of contact. The geometry and orientation of the CCNT, relative to the cantilever, were determined by analysis of a set of scanning electron micrographic images acquired at various angles. When brought into contact, the angle at the point of contact of the CCNT is $\sim 7^{\circ}$ relative to the surface normal. To change the angle, 


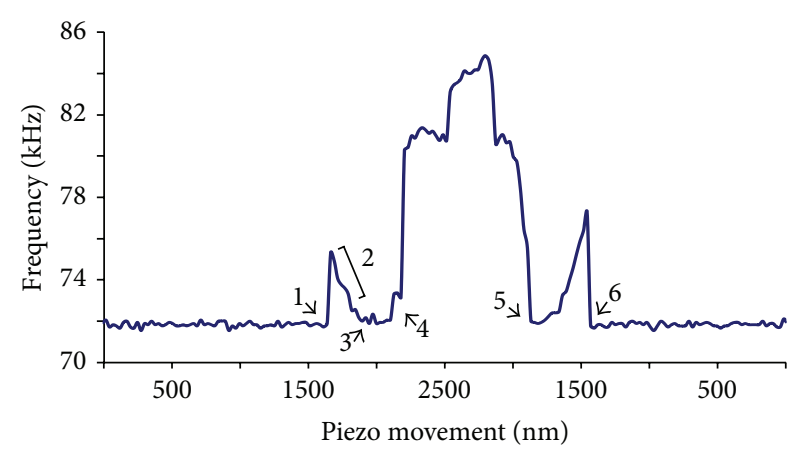

(a)

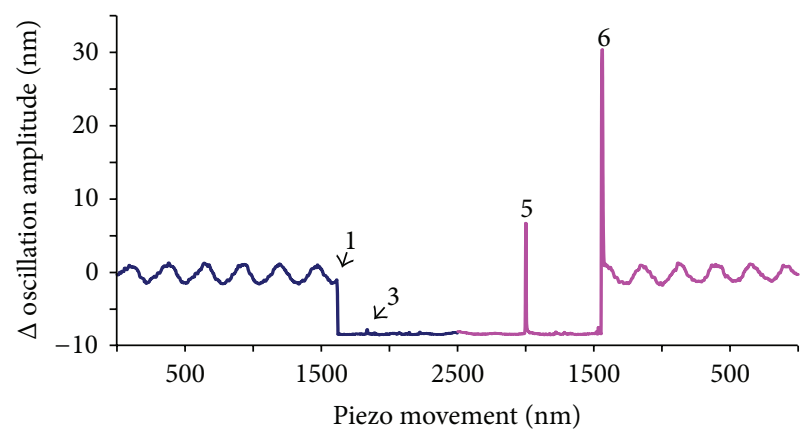

(c)

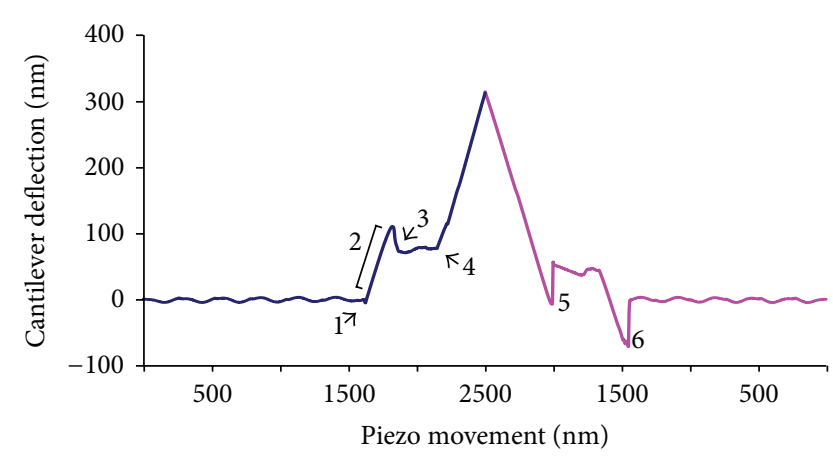

(b)

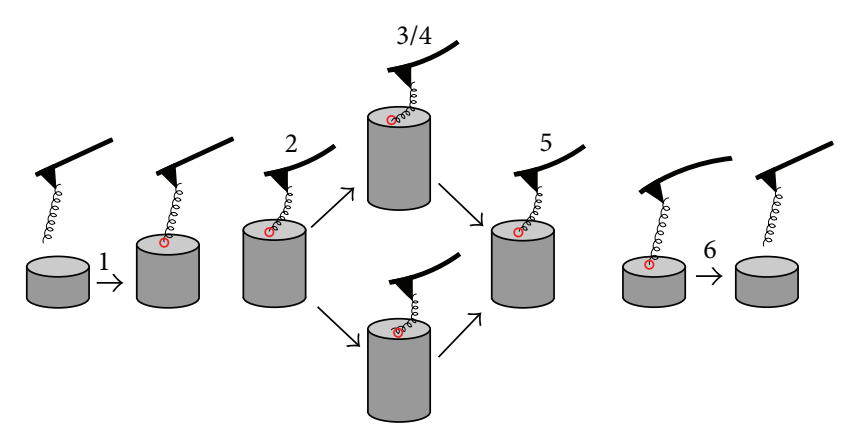

(d)

FIGURE 5: Thermal resonance frequency response (a), force distance (b), and oscillation amplitude (c) responses of the cantilever-CCNT system under $875 \mathrm{~nm}$ compression with an approach angle of $7^{\circ}$. The displayed frequency data is from the second vibration mode, due to increased $\mathrm{S} / \mathrm{N}$ response. In the plots we observe several phenomena occurring during the scanner extension: the initial contact with the substrate (1), buckling response of the nanocoil (2), and slip-stick motion $(3,4)$. During scanner retract motion we observe several ringing events in the oscillation amplitude that correlate to the return to the buckling geometry of the coil (5) and release from the surface (6).

beveled shims were used in place of standard mounting shims. Wedge-shaped shims [35] were manufactured with a $5^{\circ}$ grade and scribed to mark the reference position. A simple $180^{\circ}$ rotation of the wedge-shaped shim afforded the acquisition of data sets at nanocoil point of contact angles of $2^{\circ}$ and $12^{\circ}$. Experiments were conducted using the wedgeshaped shim in the same manner as with a flat shim. If the onset of the second jump in frequency was due to a stick event following slip, then our expectation was that this event would occur at lower scanner extension values with increasing point of contact angle.

Figure 6 is a compilation of the primary (panels (a)-(d)) and secondary (panels $(\mathrm{e})-(\mathrm{h})$ ) resonance mode frequency shifts observed during nanocoil compression at the following point of contact angles: $2^{\circ}$ (blue), $7^{\circ}$ (red), and $12^{\circ}$ (green). Panels (a) and (d) show no discernible differences in the frequency response at the three angles when the CCNT was in contact with the substrate for a scanner extension distance of only $250 \mathrm{~nm}$. At an in-contact distance of $375 \mathrm{~nm}$ (panels (b) and (f)), the frequency response on the $2^{\circ}$ and $7^{\circ}$ substrates are the same; however the occurrence of a second frequency shift is observed on the $12^{\circ}$ substrate. At greater in-contact distances (panels (c), (d), (g), and (h)), the second frequency shift event occurs at all three angles. It is interesting to note that as the contact angle is increased, the frequency response of the system during the approach and retract segments is asymmetric and correlates with point of contact angle. On the $2^{\circ}$ substrate at $875 \mathrm{~nm}$ of extension, the first and second frequency shifts have a strong symmetry with the cyclic motion of the piezo scanner. As the angle is increased to $7^{\circ}$ or $12^{\circ}$, this symmetry is lost and is indicative of increased areas of contact between the CCNT and the substrate facilitated by the slip-stick event.

Figure 7(a) presents force curve and frequency response data when the CCNT was brought in and out of contact with the substrate at an approach angle of $2^{\circ}$ for a compression distance of $375 \mathrm{~nm}$. Note that over the time interval of 23.5$25 \mathrm{~s}$, the scanner is extending without a significant change in either cantilever deflection or resonant frequency. Similarly, over the time interval 25.5-27 s, the scanner is retracting with no significant change in cantilever deflection or resonant frequency. We suggest that during each of these periods, the CCNT is sliding across the surface into a new geometry. Figure 7 (b) compares the resonant frequency response to the apparent spring constant of the cantilever-nanocoil system calculated from the thermal spectra acquired at each data point in the frequency plot. Note that over the time interval of where the frequency response is constant, the spring constant of the system oscillates. This is not indicative of a mechanical stiffening of the nanocoil but rather covariance between the baseline amplitude and the spring constant in fitting the function to the amplitude-frequency data. As the nanocoil undergoes the "slip" portion of slip stick across the surface it is under a constant force from the cantilever, rather 

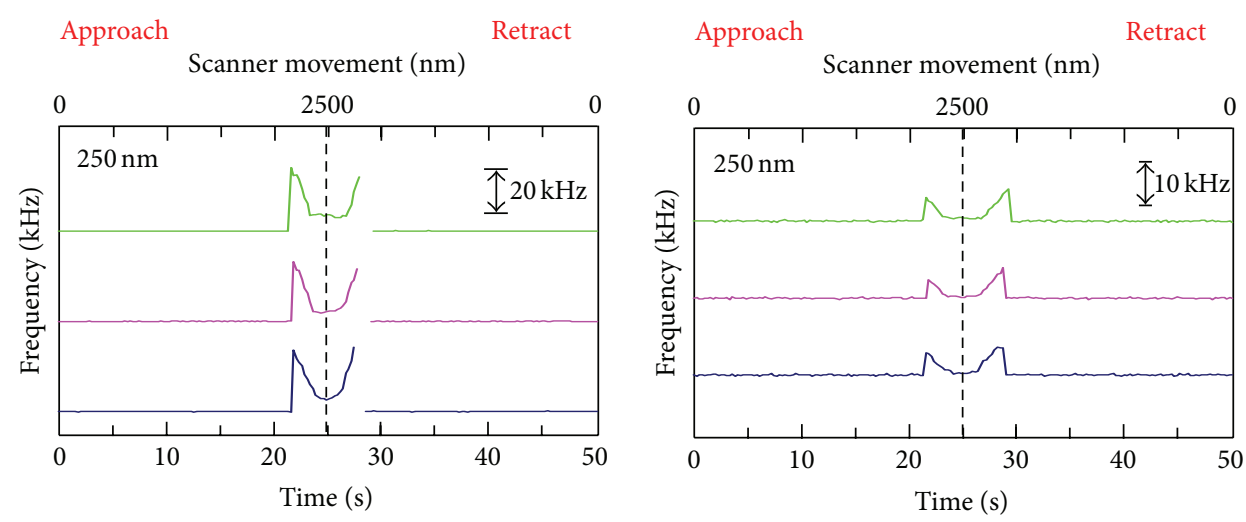

(a)

(e)
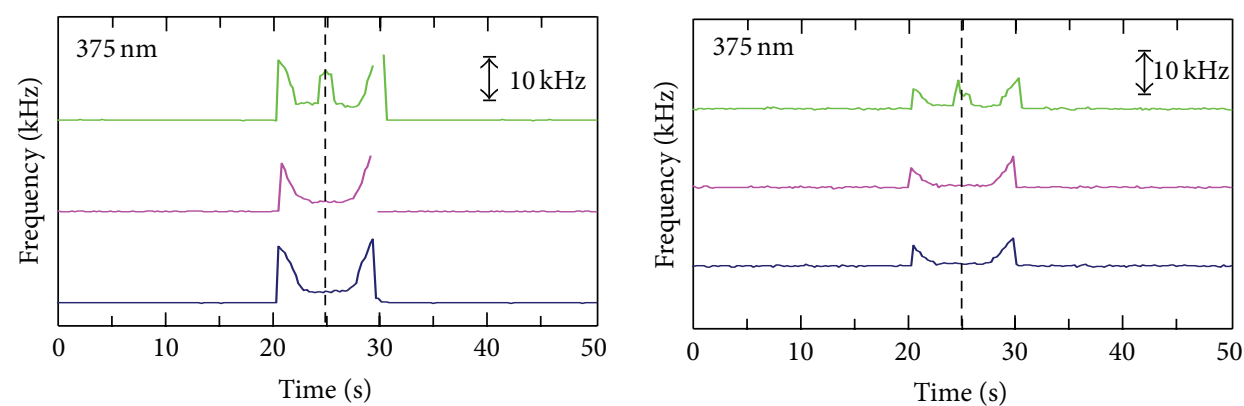

(b)

(f)
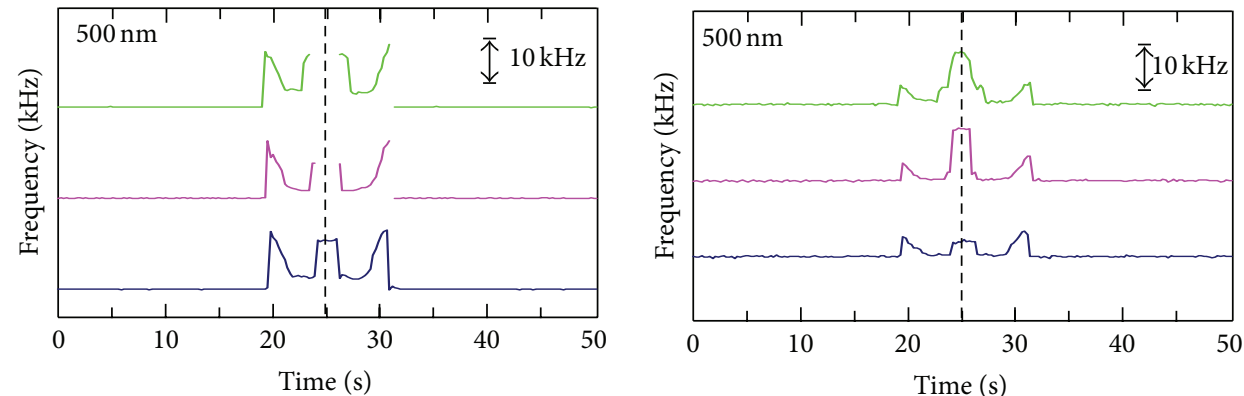

(c)

(g)

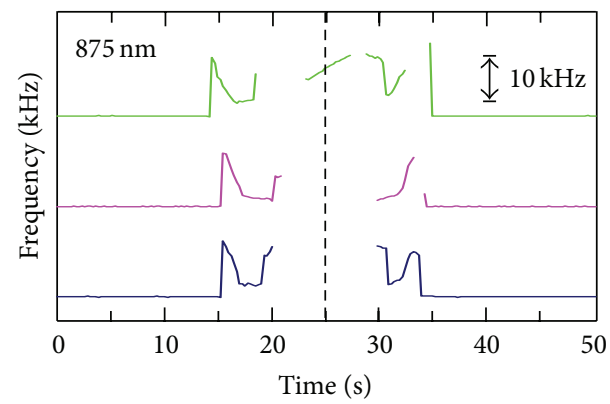

(d)

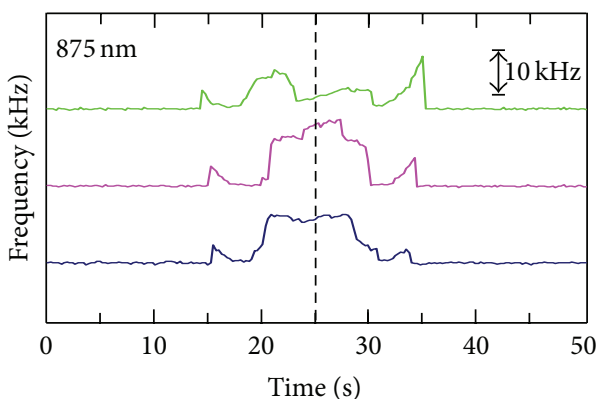

(h)

FIGURE 6: Thermal resonance frequency responses; primary mode (panels (a)-(d)) and secondary mode (panels (e)-(f)) of the CCNT at different approach angles are displayed at compression distances of $250,375,500$, and $875 \mathrm{~nm}$. The approach angle was changed from $12^{\circ}$ (green), $7^{\circ}$ (magenta), and $2^{\circ}$ (blue) through the use of an angled substrate. The z-piezo scan range was $2500 \mathrm{~nm}$; cycles were acquired at a scan rate of $0.02 \mathrm{~Hz}$. 


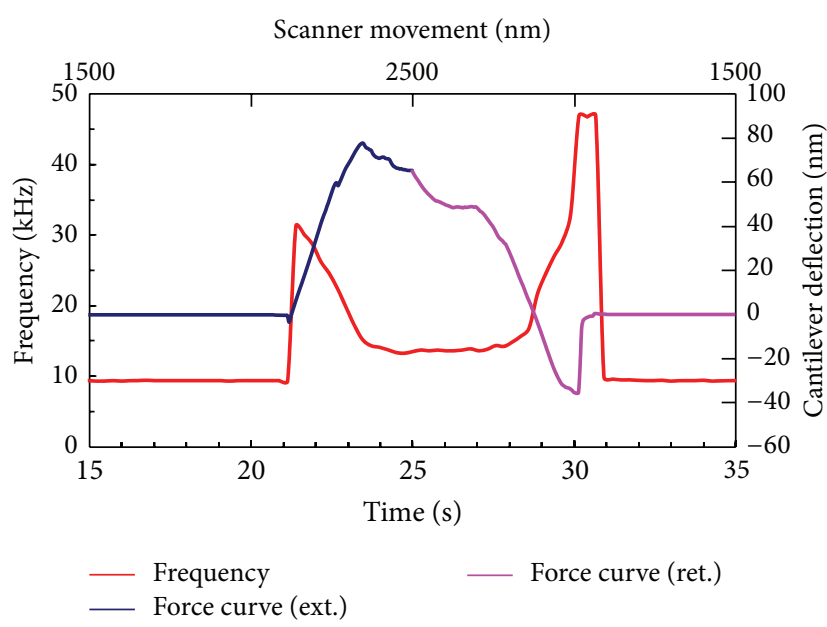

(a)

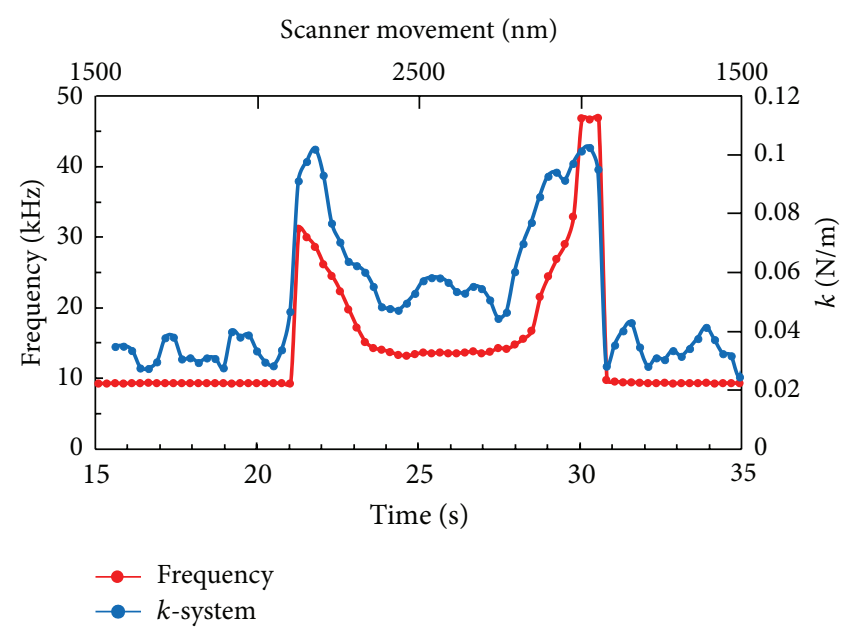

(b)

FIGURE 7: Thermal resonance frequency response with force distance (a) and calculated system stiffness (b). The data is displayed for the CCNT under a compression distance of $375 \mathrm{~nm}$ and approach angle of $2^{\circ}$ to the surface.

than a progressive force observed during buckling. As the contact area of the nanocoil increases, the frictional forces acting on the coil increase, causing the measured stiffness to increase.

The strength of adhesion depended upon the angle of compression. Adhesion forces of $1.6 \pm 0.3,1.6 \pm 0.2$, and $3.2 \pm 0.5 \mathrm{nN}$ were computed from the measured downward deflection of the system at the point of release at $2^{\circ}, 7^{\circ}$, and $12^{\circ}$, respectively. The increase in average adhesion force at $12^{\circ}$ is consistent with the increase in contact area resulting from slip-stick motion of the nanocoil at this compression angle.

TEM imaging was used to determine the internal structure for each CCNT investigated herein. Images were acquired at the point of attachment of the tube to the tip, at the free end of the tube, and at several points in between. Based on the image features at each of these locations, the nanocoils were divided into three classifications. Images of CCNTs classified as multiwalled nanocoils contained the characteristic image feature of crystalline, concentric graphitic planes. For some of the CCNTs, images near the point of attachment were devoid of features consistent with multiwalled nanotubes. The observed features were consistent with the region being either amorphous or fibrous and were most likely created during the arc discharge attachment process. For many of the CCNTs, images taken in between the point of attachment and the end revealed the crystalline structure of concentric graphitic planes with occasional defects. The presence of occasional defects along the nanocoil is both expected and a likely outcome of the CVD growth method used to prepare the CCNTs. In some instances, a MWCNT with crystalline material filling its void space was observed; CCNTs with this structural feature were classified as "filled-tube" nanocoils. In other instances, images of CCNTs revealed little or no crystalline MWNT characteristics. These CCNTs were designated as "amorphous carbon". Typical TEM images of each morphology are presented in Figure 8.

In addition to determining the internal structure, TEM images also provided measures of the coil diameter, tube diameter, wall thickness, and inner diameter of the hollow region. The number of walls was estimated dividing the difference between the outer and inner diameters of the tube with $3.4 \AA$, the thickness of a graphitic layer. Table 2 displays the TEM measurements of the nanocoils used in this study.

\section{Discussion}

A measure of the nanocoil's response to compression is its elastic modulus, $E$. To calculate this quantity from the data obtained required us to model the nanocoil as a compression spring. The spring constant for a coil $(k)$ is related to the shear modulus of the spring material through the following geometric relationship:

$$
k_{\text {fiber }}=\frac{G d^{4}}{8 D^{3} n},
$$

where $G$ is the shear modulus of the spring material, $d$ is the diameter of a filled tube or amorphous carbon fiber, $D$ is the coil diameter, and $n$ is the number of active coils [41]. Rewriting this equation to incorporate a hollow spring bar or tube yields

$$
k_{\mathrm{CCNT}}=\frac{G\left(d_{o}^{4}-d_{i}^{4}\right)}{8 D^{3} n},
$$

where $d_{o}$ and $d_{i}$ are the outer and inner diameters of the concentric tubes that comprise the coiled MWNT, respectively. For elastic materials, the shear modulus is related to the elastic modulus by the following expression:

$$
G=\frac{E}{2(1+\nu)}
$$

where $v$ is the Poisson's ratio. Assuming $v=0.3$, one can write the expression in terms of the elastic modulus to give

$$
E=2.6 G=\frac{20.8 k_{\mathrm{fiber}} D^{3} n}{d^{4}}
$$


TABLE 2: Summary of CCNT structure from TEM imaging.

\begin{tabular}{|c|c|c|c|c|c|c|c|}
\hline $\begin{array}{l}\text { CCNT } \\
\text { no. }\end{array}$ & $\begin{array}{l}\text { Free length } \\
\quad(\mathrm{nm})\end{array}$ & $\begin{array}{l}\text { Coil dia. } \\
\text { (nm) }\end{array}$ & $\begin{array}{l}\text { Nanotube width } \\
\qquad(\mathrm{nm})\end{array}$ & $\begin{array}{c}\text { Wall } \\
\text { thickness } \\
(\mathrm{nm})\end{array}$ & $\begin{array}{l}\text { Inner radius } \\
(\mathrm{nm})\end{array}$ & $\begin{array}{l}\text { Outer radius } \\
\qquad(\mathrm{nm})\end{array}$ & $\begin{array}{l}\text { No. of } \\
\text { walls }\end{array}$ \\
\hline 1 & 1597 & 226 & 49.6 & 11.4 & 13.3 & 24.8 & 34 \\
\hline 2 & 3247 & 58.7 & 32.3 & 7.9 & 8.3 & 16.1 & \\
\hline 3 & 5748 & 269 & 90.4 & 37.9 & 7.2 & 45.2 & 112 \\
\hline 4 & & 175 & 71.8 & 16.6 & 19.2 & 35.9 & \\
\hline 5 & 8324 & 142 & 55.3 & 25.4 & 2.2 & 27.6 & \\
\hline 6 & & 576 & 83.9 & 31.7 & 10.2 & 42.0 & 93 \\
\hline 7 & 4756 & 181 & 58.3 & 20.4 & 8.7 & 29.1 & 60 \\
\hline 8 & 7542 & 415 & 67.5 & 17.2 & 16.5 & 33.7 & 51 \\
\hline 9 & 11360 & 120 & 63.5 & 20.8 & 10.9 & 31.8 & \\
\hline 10 & & 276 & 59.8 & 8.1 & 21.8 & 29.9 & 24 \\
\hline 11 & 3142 & 228 & 61.7 & 15.8 & 15.0 & 30.9 & 47 \\
\hline 12 & 5420 & 127 & 32.8 & 13.3 & 3.1 & 16.4 & 39 \\
\hline 13 & 4370 & 217 & 52.6 & 21.6 & 4.7 & 26.2 & 63 \\
\hline
\end{tabular}

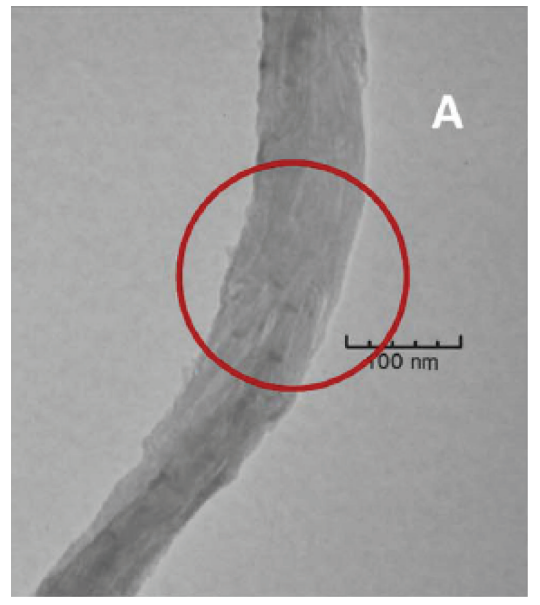

(a)

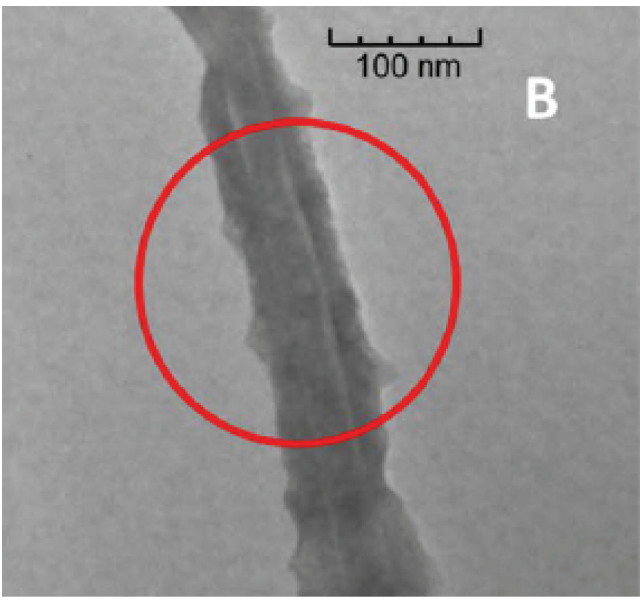

(b)

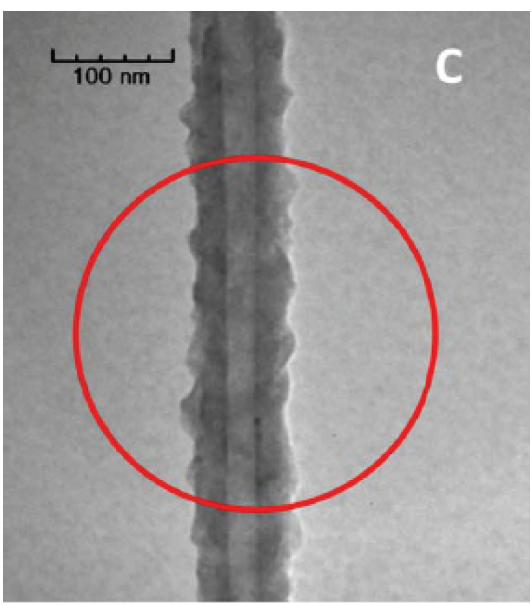

(c)

FIGURE 8: TEM images illustrating the observed morphology of (a) amorphous, (b) filled, and (c) MWNT CCNTs. 
for the filled tubes or amorphous carbon fibers and

$$
E=2.6 G=\frac{20.8 k_{\mathrm{CCNT}} D^{3} n}{\left(d_{0}^{4}-d_{i}^{4}\right)}
$$

for the coiled carbon nanotubes once the spring constant of the coil has been determined.

The stiffness of the nanocoil to axial compression is directly related to the thermal resonance frequency at the point of contact of the nanocoil with the substrate. The resonance data presented herein was collected after ensemble averaging of the power spectral densities. This averaging was necessary to improve the signal-to-noise ratio in the amplitude versus frequency data. By doing so, we lose the ability to track, with certainty, the frequency at the point of contact. If, during the time interval of data collection and ensemble averaging, the nanocoil undergoes bending, buckling, or slipstick motion, then the average frequency will be less than that at the point of contact. The bending/buckling phenomenon is facilitated by open end spring geometry [41]. Thus, the compression stiffness of the CCNT can only be estimated from the resonant frequency of the cantilever at the point of contact with the substrate surface. The spring constant of the coil is computed from the effective spring constant of the system using a springs-in-parallel model. For the CCNT upon which the data in Figure 7 was obtained, a $k_{\mathrm{CCNT}}$ of $0.106 \pm 0.003 \mathrm{Nm}^{-1}$ was computed using this approach. No significant difference in the calculated value of $k_{\mathrm{CCNT}}$ with respect to the angle at the point of contact was observed. The identical approach was used to determine the stiffness of the other CCNTs examined herein; the measured values ranged from 0.020 to $0.115 \mathrm{Nm}^{-1}$. Hayashida and coworkers [8] employed forced expansion of five different CCNTs to obtain spring constants ranging from 0.01 to $0.6 \mathrm{Nm}^{-1}$. Similarly Chen et al. [29] obtained a spring constant of $0.12 \mathrm{Nm}^{-1}$ from tensile loading of a CCNT connected between two AFM probes. Thus, our $k_{\mathrm{CCNT}}$ values lie within the range previously obtained for CCNTs but well below the values predicted from simulations [42].

The elastic modulus for each CCNT was calculated from the measured $k_{\mathrm{CCNT}}$ using either (4) or (5), depending upon the morphology of the nanocoil. Table 3 presents a comparison of the computed values with CCNT morphology. There is a large difference in the elastic modulus between tubular nanocoils and amorphous carbon nanocoils. A mean of $15 \pm 8 \mathrm{GPa}$ was found for filled and hollow nanocoils whereas a mean of $1.4 \pm 1.1 \mathrm{GPa}$ was found for the amorphous nanocoils. In contrast, filled nanocoils have a modulus of $19 \pm 10 \mathrm{GPa}(n=3)$, and the MWNT-structured coils have a modulus of $13 \pm 5 \mathrm{GPa}(n=6)$. These values are not statistically distinguishable from each other. Previously, Volodin et al. obtained much higher values (400-900 GPa) from AFM imaging studies [43]. Hayashida and coworkers obtained values ranging from 40 to $130 \mathrm{GPa}$ from tensile loading experiments [8]. But our range is in agreement with very recent simulations by Wang and coworkers [44]. They evaluated a CCNTs response to both tension and compression using classical molecular dynamics simulations. Their findings indicate that the first linear regime of the stress
TABLE 3: Comparison of CCNT elastic moduli and morphology.

\begin{tabular}{lcc}
\hline CCNT no. & Classification & $\begin{array}{c}\text { Elastic modulus } \\
(\mathrm{GPa})\end{array}$ \\
\hline 1 & Filled & 10 \\
2 & Amorphous & 2.7 \\
3 & MWNT & 12.2 \\
4 & Amorphous & 2.0 \\
5 & Amorphous & 0.6 \\
6 & MWNT & 13 \\
7 & MWNT & 6.2 \\
8 & Filled & 17.8 \\
9 & Amorphous & 0.4 \\
10 & Filled & 31.4 \\
11 & MWNT & 22.9 \\
12 & MWNT & 9.3 \\
13 & MWNT & 11.8 \\
\hline
\end{tabular}

versus strain curve for the CCNT under compression extends from zero to $9.2 \mathrm{GPa}$ at a strain of $16 \%$. They predict that as long as the strain is less than this value, the deformation of the CCNT is reversible. Reversible deformation is observed in the repeated loading depicted in Figure 2.

\section{Summary}

MDFS involves the simultaneous acquisition of the cantilever's deflection and thermal resonance data continuously throughout the acquisition of a force curve. We have demonstrated herein that when this technique is applied to the compression of a CCNT, unique signatures for buckling, bending, and slip-stick events of the nanocoil are observed. These mechanical responses to applied load are sometimes difficult to identify with conventional force spectroscopy (i.e., force or oscillation amplitude curves). Spring constants for the CCNT have been determined from thermal resonance frequency shifts during compression. Modeling of the CCNTs as compression springs has afforded estimates of their elastic modulus; these estimates fall within the theoretically predicted range and correlate with the nanocoil's morphology. From the results presented herein, we conclude that the major structural factor determining the mechanical properties of CCNTs is whether the core is amorphous or consists of multiwalled nanotubes.

\section{Conflict of Interests}

The authors declare that there is no conflict of interests regarding the publication of this paper.

\section{Acknowledgments}

The authors acknowledge fruitful discussions with Professor Jonathan Colton and assistance in data analysis by Miss Kelsey Beavers. Research collaboration with Ames Research Center was conducted under the guidance of Dr. Cattien V. 
Nguyen. Financial support from the NASA Graduate Student Researchers Program is gratefully acknowledged.

\section{References}

[1] A. A. Lucas, P. Lambin, V. Ivanov, and J. B. Nagy, "Coiled carbon nanotubes," in Proceedings of the 2nd International Winterschool on Electronic Properties of Novel Materials, Progress in Fullerene Research, pp. 122-125, 1994.

[2] X. B. Zhang, X. F. Zhang, D. Bernaerts et al., "The texture of catalytically grown coil-shaped carbon nanotubules," Europhysics Letters, vol. 27, no. 2, pp. 141-146, 1994.

[3] V. Celorrio, L. Calvillo, M. V. Martínez-Huerta, R. Moliner, and M. J. Lázaro, "Study of the synthesis conditions of carbon nanocoils for energetic applications," Energy and Fuels, vol. 24, no. 6, pp. 3361-3365, 2010.

[4] D. Fejes and K. Hernadi, "A review of the properties and CVD synthesis of coiled carbon nanotubes," Materials, vol. 3, no. 4, pp. 2618-2642, 2010.

[5] M. J. Hanus and A. T. Harris, "Synthesis, characterisation and applications of coiled carbon nanotubes," Journal of Nanoscience and Nanotechnology, vol. 10, no. 4, pp. 2261-2283, 2010.

[6] A. Csató, A. Szabó, A. Fonseca et al., "Synthesis and characterisation of coiled carbon nanotubes," Catalysis Today, vol. 181, no. 1, pp. 33-39, 2012.

[7] D. Li, L. Pan, Y. Wu, and W. Peng, "The effect of changes in synthesis temperature and acetylene supply on the morphology of carbon nanocoils," Carbon, vol. 50, no. 7, pp. 2571-2580, 2012.

[8] T. Hayashida, L. Pan, and Y. Nakayama, "Mechanical and electrical properties of carbon tubule nanocoils," Physica B, vol. 323, no. 1-4, pp. 352-353, 2002.

[9] A. F. Da Fonseca and D. S. Galvão, "Mechanical properties of nanosprings," Physical Review Letters, vol. 92, no. 17, Article ID 175502, 4 pages, 2004.

[10] W. M. Huang, "Mechanics of coiled nanotubes in uniaxial tension," Materials Science and Engineering A, vol. 408, no. 1-2, pp. 136-140, 2005.

[11] K.-I. Nakamatsu, M. Nagase, H. Namatsu, and S. Matsui, "Mechanical characteristics of diamond-like-carbon nanosprings fabricated by focused-ion-beam chemical vapor deposition," Japanese Journal of Applied Physics, Part 2, vol. 44, no. 37-41, pp. L1228-L1230, 2005.

[12] H. Bi, K. C. Kou, K. Ostrikov, J. Q. Zhang, and Z. C. Wang, "Mechanical model and superelastic properties of carbon microcoils with circular cross-section," Journal of Applied Physics, vol. 106, no. 2, Article ID 023520, 2009.

[13] K. T. Lau, M. Lu, H. L. Li, L. M. Zhou, and D. Hui, "Heat absorbability of single-walled, coiled and bamboo nanotube/epoxy nano-composites," Journal of Materials Science, vol. 39, no. 18, pp. 5861-5863, 2004.

[14] S. Motojima, T. Muraki, T. Suzuki et al., "Preparation of ceramics/carbon microcoils composites using carbon microcoils as a template," Transactions of the Materials Research Society Of Japan, vol. 29, no. 2, pp. 465-468, 2004.

[15] A. Volodin, D. Buntinx, M. Ahlskog, A. Fonseca, J. B. Nagy, and C. van Haesendonck, "Coiled carbon nanotubes as self-sensing mechanical resonators," Nano Letters, vol. 4, no. 9, pp. 17751779, 2004.

[16] M. Lu, K.-T. Lau, J.-C. Xu, and H.-L. Li, "Coiled carbon nanotubes growth and DSC study in epoxy-based composites," Colloids and Surfaces A, vol. 257-258, pp. 339-343, 2005.
[17] K.-T. Lau, C. Gu, and D. Hui, "A critical review on nanotube and nanotube/nanoclay related polymer composite materials," Composites B, vol. 37, no. 6, pp. 425-436, 2006.

[18] K. T. Lau, M. Lu, and D. Hui, "Coiled carbon nanotubes: synthesis and their potential applications in advanced composite structures," Composites B, vol. 37, no. 6, pp. 437-448, 2006.

[19] X.-F. Li, K.-T. Lau, and Y.-S. Yin, "Nano-mechanical properties of coiled carbon nanotube reinforced epoxy composites," Key Engineering Materials, vol. 334-335, pp. 673-676, 2007.

[20] A. Leela Mohana Reddy, R. I. Jafri, N. Jha, S. Ramaprabhu, and P. M. Ajayan, "Carbon nanocoils for multi-functional energy applications," Journal of Materials Chemistry, vol. 21, no. 40, pp. 16103-16107, 2011.

[21] R. B. Rakhi, W. Chen, and H. N. Alshareef, "Conducting polymer/carbon nanocoil composite electrodes for efficient supercapacitors," Journal of Materials Chemistry, vol. 22, no. 11, pp. 5177-5183, 2012.

[22] M. A. Poggi, J. S. Boyles, L. A. Bottomley et al., "Measuring the compression of a carbon nanospring," Nano Letters, vol. 4, no. 6, pp. 1009-1016, 2004.

[23] K. M. Barker, A. Ferri, and L. A. Bottomley, "Adhesive and mechanical properties of carbon nanotube probes contacting chemically-treated surfaces," ASME Conference Proceedings, vol. 2011, pp. 739-746, 2011.

[24] K. M. Barker, M. A. Poggi, L. Lizarraga, P. T. Lillehei, A. A. Ferri, and L. A. Bottomley, "Peeling of long, straight carbon nanotubes from surfaces," Journal of Nanotechnology. In press.

[25] B. Bhushan and X. Ling, "Adhesion and friction between individual carbon nanotubes measured using force-versus-distance curves in atomic force microscopy," Physical Review B, vol. 78, no. 4, Article ID 045429, 9 pages, 2008.

[26] B. Bhushan, X. Ling, A. Jungen, and C. Hierold, "Adhesion and friction of a multiwalled carbon nanotube sliding against singlewalled carbon nanotube," Physical Review B, vol. 77, no. 16, Article ID 165428, 12 pages, 2008.

[27] J. Buchoux, J.-P. Aimé, R. Boisgard, C. V. Nguyen, L. Buchaillot, and S. Marsaudon, "Investigation of the carbon nanotube AFM tip contacts: free sliding versus pinned contact," Nanotechnology, vol. 20, no. 47, Article ID 475701, 2009.

[28] J. Buchoux, L. Bellon, S. Marsaudon, and J.-P. Aimé, “Carbon nanotubes adhesion and nanomechanical behavior from peeling force spectroscopy," European Physical Journal B, vol. 84, no. 1, pp. 69-77, 2011.

[29] X. Chen, S. Zhang, D. A. Dikin et al., "Mechanics of a carbon nanocoil," Nano Letters, vol. 3, no. 9, pp. 1299-1304, 2003.

[30] M. R. Falvo, G. J. Clary, R. M. Taylor II et al., "Bending and buckling of carbon nanotubes under large strain," Nature, vol. 389, no. 6651, pp. 582-584, 1997.

[31] M. Ishikawa, M. Yoshimura, and K. Ueda, "A study of friction by carbon nanotube tip," Applied Surface Science, vol. 188, no. 3-4, pp. 456-459, 2002.

[32] T. Yonemura, Y. Suda, H. Tanoue et al., "Torsion fracture of carbon nanocoils," Journal of Applied Physics, vol. 112, no. 8, Article ID 084311, 4 pages, 2012.

[33] R. M. D. Stevens, N. A. Frederick, B. L. Smith, D. E. Morse, G. D. Stucky, and P. K. Hansma, "Carbon nanotubes as probes for atomic force microscopy," Nanotechnology, vol. 11, no. 1, pp. 1-5, 2000.

[34] M. Hegner, P. Wagner, and G. Semenza, "Ultralarge atomically flat template-stripped $\mathrm{Au}$ surfaces for scanning probe microscopy," Surface Science, vol. 291, no. 1-2, pp. 39-46, 1993. 
[35] M. J. D’Amato, M. S. Marcus, M. A. Eriksson, and R. W. Carpick, "Phase imaging and the lever-sample tilt angle in dynamic atomic force microscopy," Applied Physics Letters, vol. 85, no. 20, pp. 4738-4740, 2004.

[36] J. L. Hutter and J. Bechhoefer, "Calibration of atomic-force microscope tips," Review of Scientific Instruments, vol. 64, no. 7, pp. 1868-1873, 1993.

[37] M. A. Poggi, L. A. Bottomley, and P. T. Lillehei, "Measuring the adhesion forces between alkanethiol-modified AFM cantilevers and single walled carbon nanotubes," Nano Letters, vol. 4, no. 1, pp. 61-64, 2004.

[38] M. A. Poggi, P. T. Lillehei, and L. A. Bottomley, "Chemical force microscopy on single-walled carbon nanotube paper," Chemistry of Materials, vol. 17, no. 17, pp. 4289-4295, 2005.

[39] C.-L. Zhang and H.-S. Shen, "Buckling and postbuckling analysis of single-walled carbon nanotubes in thermal environments via molecular dynamics simulation," Carbon, vol. 44, no. 13, pp. 2608-2616, 2006.

[40] G. A. Tomlinson, "A molecular theory of friction," Philosophical Magazine, vol. 7, no. 46, pp. 905-939, 1929.

[41] A. M. Wahl, Mechanical Springs, McGraw-Hill, New York, NY, USA, 1963.

[42] S. H. Ghaderi and E. Hajiesmaili, "Molecular structural mechanics applied to coiled carbon nanotubes," Computational Materials Science, vol. 55, pp. 344-349, 2012.

[43] A. Volodin, M. Ahlskog, E. Seynaeve, C. van Haesendonck, A. Fonseca, and J. B. Nagy, "Imaging the elastic properties of coiled carbon nanotubes with atomic force microscopy," Physical Review Letters, vol. 84, no. 15, pp. 3342-3345, 2000.

[44] J. Wang, T. Kemper, T. Liang, and S. B. Sinnott, "Predicted mechanical properties of a coiled carbon nanotube," Carbon, vol. 50, no. 3, pp. 968-976, 2012. 

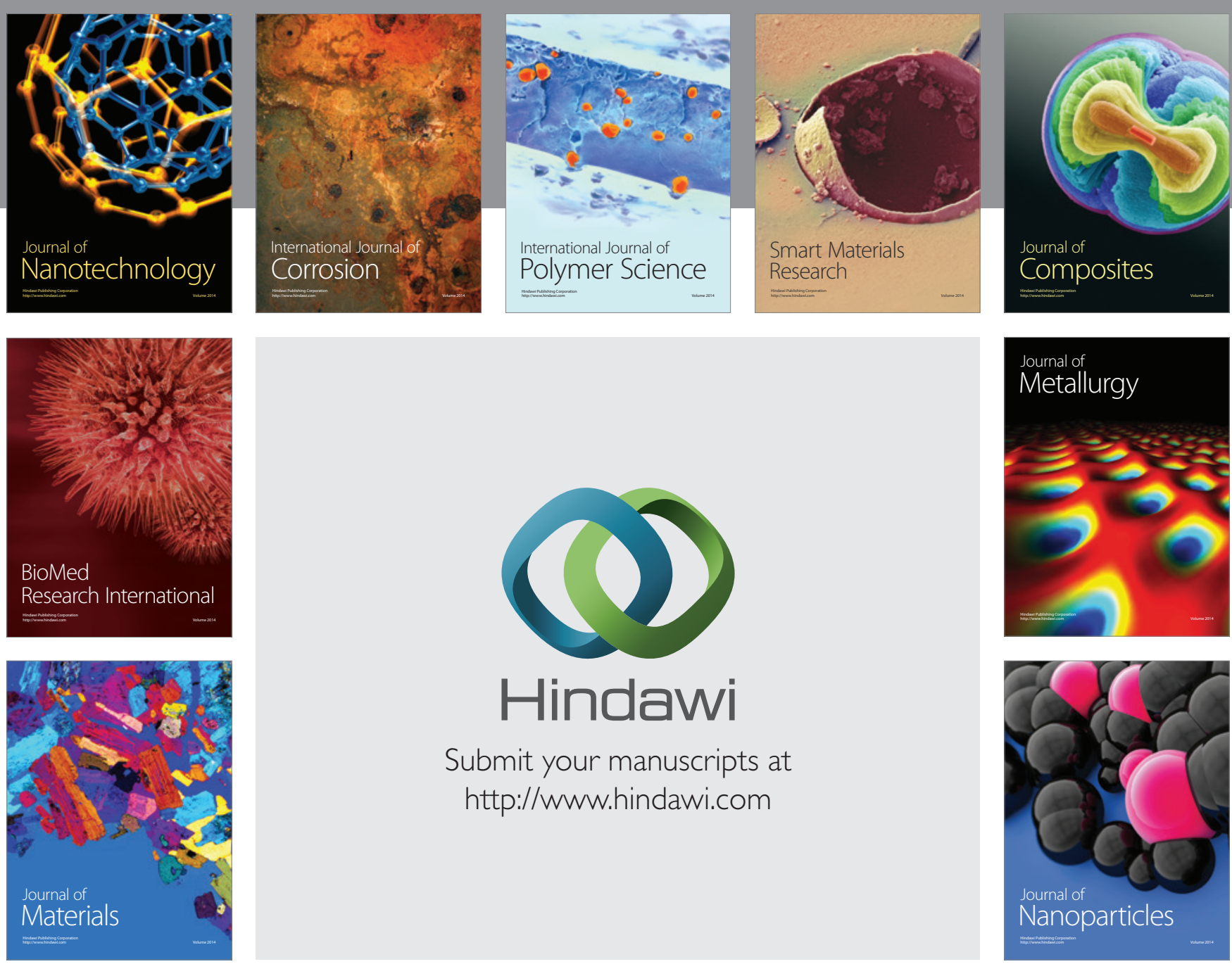

Submit your manuscripts at http://www.hindawi.com
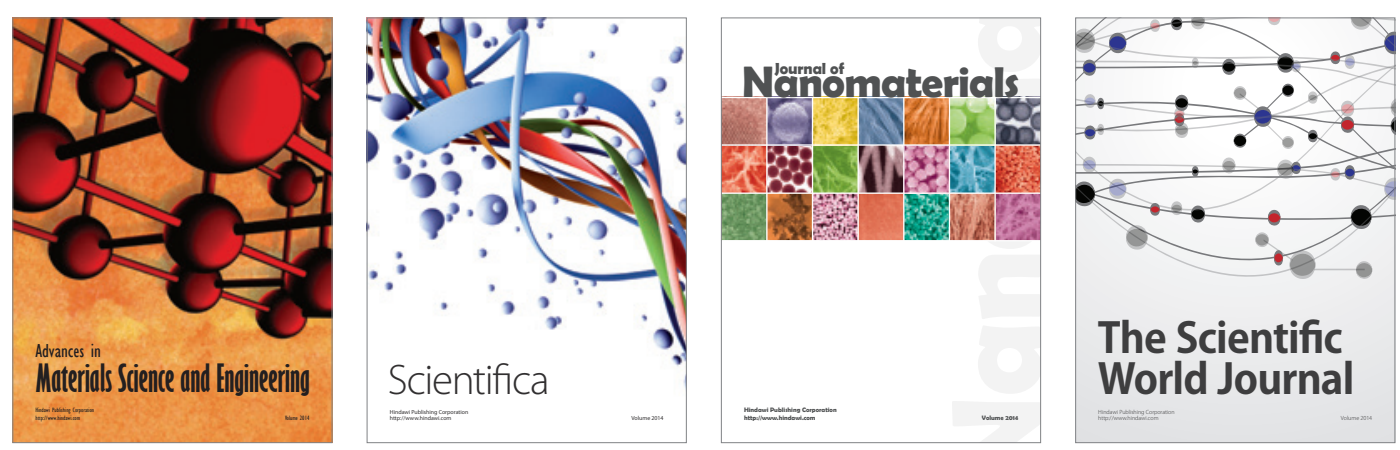

\section{The Scientific World Journal}
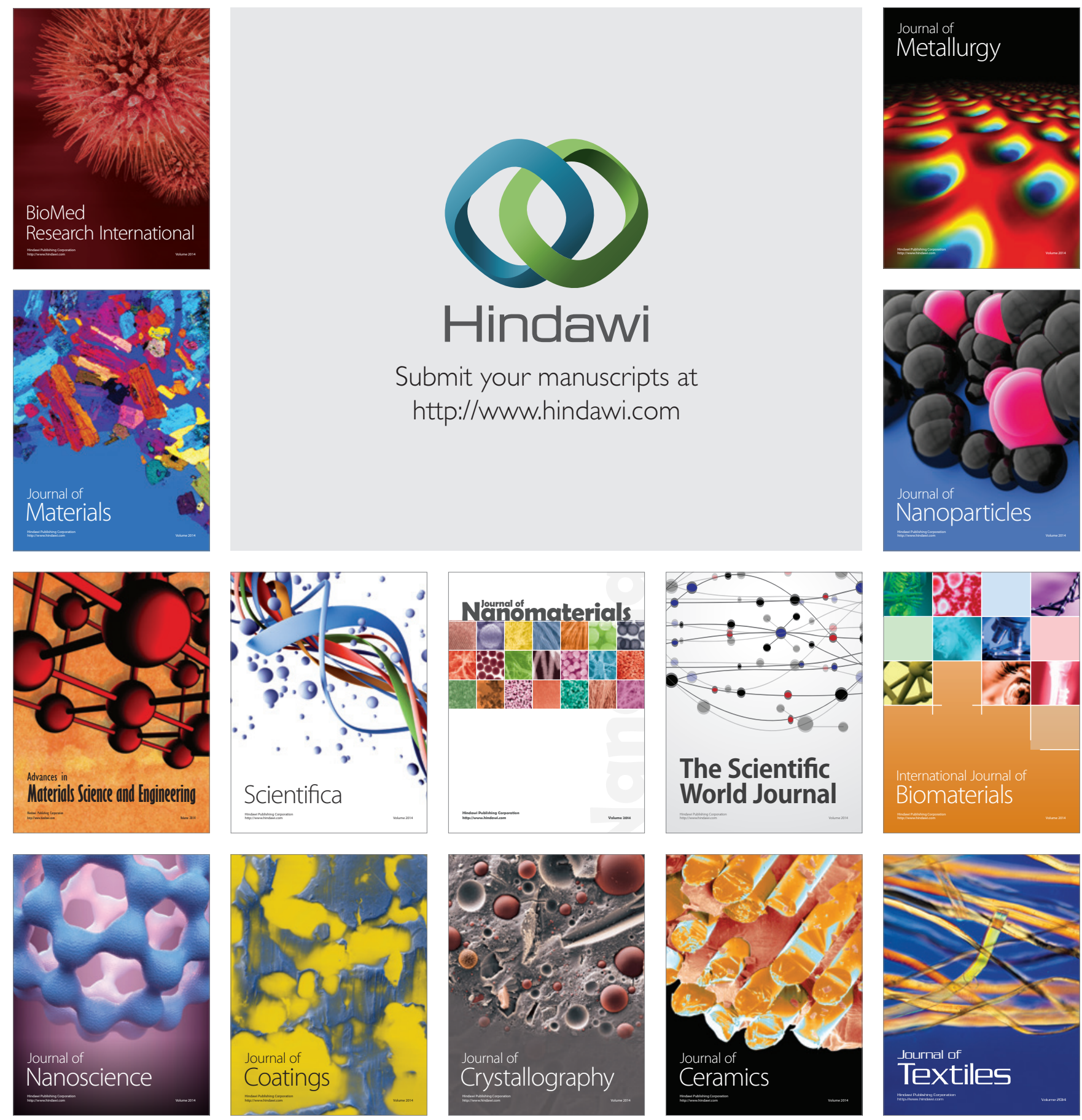